\title{
Off-axis short GRBs from structured jets as counterparts to GW events
}

\author{
Adithan Kathirgamaraju $\mathbf{u}^{* \dagger}$ \\ Department of Physics and Astronomy, Purdue University, 525 Northwestern Avenue, West \\ Lafayette, IN 47907, USA \\ E-mail: akathirg@purdue.edu
}

\section{Rodolfo Barniol Duran}

Department of Physics and Astronomy, California State University, Sacramento, 6000 J Street,

Sacramento, CA 95819, USA

E-mail: barniolduran@csus.edu

\section{Dimitrios Giannios}

Department of Physics and Astronomy, Purdue University, 525 Northwestern Avenue, West Lafayette, IN 47907, USA

E-mail: dgiannio@purdue.edu

\begin{abstract}
Binary neutron star mergers are considered to be the most favorable sources that produce electromagnetic (EM) signals associated with gravitational waves (GWs). They are also the likely progenitors of short duration gamma-ray bursts (GRBs). The brief gamma-ray emission (the "prompt" GRB emission) is produced by ultra-relativistic jets, as a result, it is strongly beamed over a small solid angle along the jet. As a result is estimated to be a decade or so before a short GRB jet within the LIGO volume points along our line of sight. However, we argue that for a realistic jet model, one whose luminosity and Lorentz factor vary smoothly with angle, the prompt signal can be detected for a significantly broader range of viewing angles. This can lead to an "off-axis" short GRB as an EM counterpart. Our estimates and simulations show, that with the aid of the temporal coincidence from a LIGO trigger, it is feasible to detect these prompt signals with a detector such as Fermi, even if the observer is substantially misaligned with respect to the jet.
\end{abstract}

7th Fermi Symposium 2017

15-20 October 2017

Garmisch-Partenkirchen, Germany

\footnotetext{
*Speaker.

${ }^{\dagger}$ The complete publication for this work can be found under MNRAS Letters [14], DOI:10.1093/mnrasl/slx175
} 


\section{Introduction}

Double neutron star (NS-NS) mergers (or neutron star-black hole mergers) are the most promising source of gravitational waves (GWs) and accompanying electromagnetic (EM) signals e.g., [1]. Such mergers are detectable by LIGO up to a few hundreds of Mpc [2] These mergers are also considered to be the most likely source of short GRBs e.g., [3-5].

The "prompt" $\gamma$-ray emission from short GRBs is believed to be strongly beamed along an ultra-relativistic jets with half opening angle $\theta_{\mathrm{j}}$ and Lorentz factor $\Gamma_{\text {core }} \gtrsim 30$ [4] . If $\Gamma_{\text {core }} \theta_{j}>1$, it will be extremely difficult to detect the prompt emission from a short GRB jet that is misaligned by an angle $\theta>\theta_{\mathrm{j}}$ with respect to Earth. In fact, the observed rate of short GRBs indicates that it will be a decade or more before the luminous core of a GRB jet points along our line of sight within the LIGO detectability volume of neutron star mergers e.g., [6]. However, in a realistic jet model, we expect the Lorentz factor and luminosity of the jet to vary smoothly with angle, resulting in a fast, luminous core at smaller angles, and a slower fainter "sheath" at larger angles, see Fig. 1. This has also been motivated by quite recent numerical [7, 8] and theoretical [9] investigations of magnetohydrodynamic (MHD) jet models. Even though the prompt emission from the extended lateral "sheath" is much fainter than a typical on-axis short GRB, we propose that, due to the proximity of a short GRB within the LIGO volume of binary neutron star mergers ( $200 \mathrm{Mpc})$, and with the aid of a coincident LIGO trigger, this faint emission can make for a detectable source. This can lead to an "off-axis" short GRB as an EM counterpart to GW events.

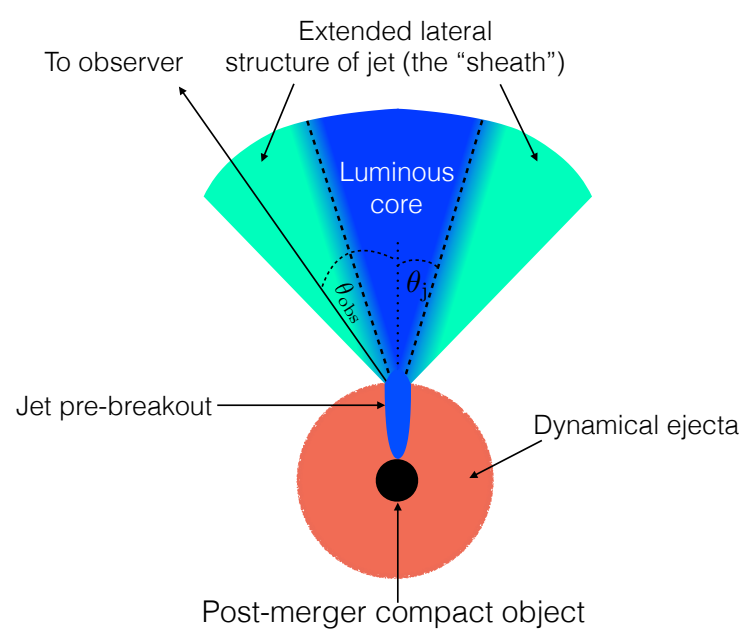

Figure 1: A schematic of a short GRB jet. The prompt emission from the jet's luminous core (routinely observed as a short GRB) is strongly beamed and can only be detected by observers located within $\theta_{\mathrm{j}}$ from the jet axis. However, the jet is expected to have a lateral structure that moves slower and is fainter than the luminous core. Given the proximity of a LIGO-triggered short GRB, Fermi and Swift can potentially detect the prompt emission from this lateral structure even if the jet is misaligned with respect to our line of sight (see Section 3). 


\section{The structure of the jet}

We perform relativistic MHD simulations using the HARM code [10] in order to investigate the structure of the jet, i.e., how the Lorentz factor and power of the jet depends on the polar angle. The jet is launched with magnetization $\mu=2 p_{\text {mag, }, 0} / \rho_{0} c^{2} \simeq 25$, where $p_{\text {mag }, 0}$ is the magnetic pressure and $\rho_{0}$ is the density at the base of the jet. Fig. 2 shows the density and Lorentz factor contour plots for a snapshot of the simulation. Very close to the compact object, we set a high density to mimic the post-merger dynamical ejecta [11, 12]. For more details on the numerical setup, see $[13,14]$. From the simulations, we extract the Lorentz factor and power of the jet as a function of polar angle along a fixed radius (Fig 3), and use it to calculate the prompt emission an observer can expect to receive from this structured jet.
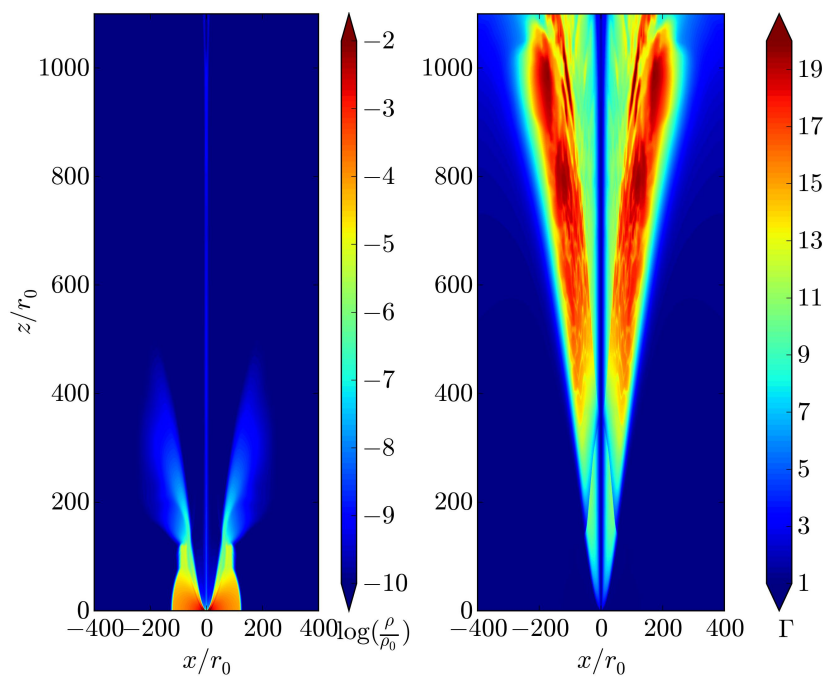

Figure 2: Numerical simulation of the jet showing 2D cuts of density (left panel) and Lorentz factor (right panel), where $r_{0}$ stands for a few times the radius of the central compact object. The jet accelerates as it breaks out from the dynamical ejecta and spread sideways. At large distances the jet turns conical and its lateral structure is fixed.

\section{Emission profile of a structured jet}

We calculate the observed luminosity from the jet by assuming a fixed fraction of the jet power (shown in Fig. 3) is radiated isotropically in the co-moving frame of the jet (see [14] for more details on the calculation). Fig. 4 shows the observed (radiated) luminosity, normalized to peak, as a function of observer angle for two types of jet models. The blue line (labeled as structured) shows the emission profile from the structured jet obtained from our simulations. The orange line (labeled uniform) shows the emission profile for a uniform or top-hat jet model, which is a commonly invoked model for GRB jets. The uniform jet model assumes a constant power and Lorentz factor within the jet, which extends up to a half opening angle $\theta_{\mathrm{j}}$, and the jet abruptly vanishes for angles larger than $\theta_{\mathrm{j}}$. In order to make a fairer comparison of the emission profiles between the two jet models, we assume the uniform jet has $\Gamma=20$, which corresponds to the peak Lorentz factor of our 


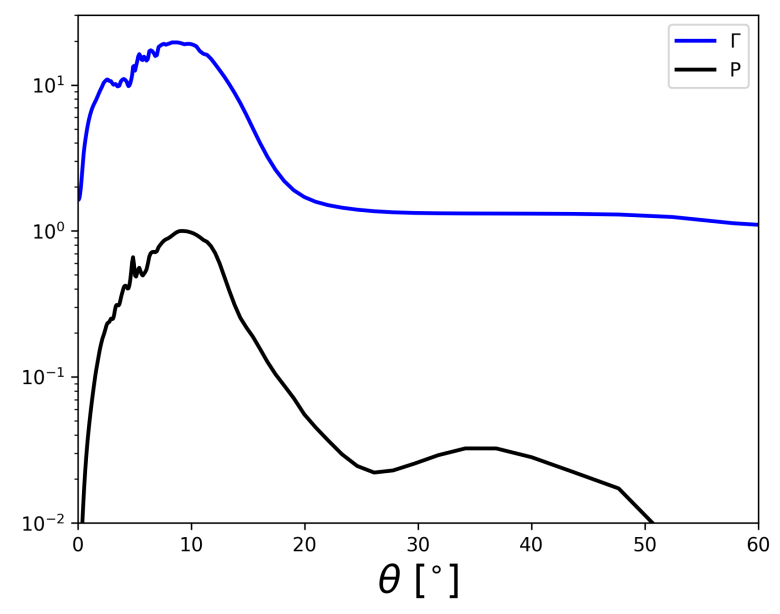

Figure 3: Jet power per steradian, $P$ (arbitrary units), and jet Lorentz factor $\Gamma$ as a function of polar angle $\theta$ with respect to the jet axis, along a fixed radius of $800 r_{0}, r_{0}$ is a few times the radius of the compact object.

structured jet and $\theta_{\mathrm{j}}=10^{\circ}$, which signifies the extent of the luminous core of our structured jet (see Fig. 3). We can estimate a delay time for this emission by calculating the photospheric radius of the jet $r_{\mathrm{ph}}=L \sigma_{T} / 4 \pi \Gamma^{2} \mu m_{p} c^{3}$, where $\sigma_{T}$ is the Thomson cross-section and $m_{p}$ is the proton mass. We estimate that even for significantly misaligned observers $\left(\theta_{\text {obs }} \gtrsim 40^{\circ}\right)$, the delay will be $\sim$ few to few tens of seconds after the merger, making for a prompt EM signal.

The typical observed short GRBs occurring at cosmological distances are viewed on-axis. If we scaled the count rate of those short GRBs to a distance of $\sim 200$ Mpc (LIGO detectability volume), it would result in an extremely high count rate of $\sim 10^{6}$ photon/s. From a previous analysis [15], it is estimated that with the aid of a coincident LIGO trigger, a count rate of $\sim 10^{3}$ photons/s is required in order to make a robust short GRB detection associated with the GW event (using a detector such as Fermi GBM). If we were to put these estimates in Fig. 4, the peak would correspond to $10^{6}$ photons/s and the detection limit of $10^{3}$ photons/s would correspond to a value of $10^{-3}$ when normalized to the peak, this limit is indicated by the dashed gray line in Fig. 4. From this figure, we determine that the emission from a structured jet can be detected for a much larger range of observing angles when compared to the emission of a uniform jet. This can increase the chances of detecting a short GRB that is coincident with a GW event. As an estimate, the beaming corrected rate of short GRBs within the LIGO detectability volume of NS-NS mergers is $\sim 10$ per year [16]. Guided by Fig. 4, we assume such a short GRB is detectable up to a viewing angle of $60^{\circ}$ and obtain a detection rate of a few short GRBs per year. To clarify, this is the rate of detecting a short GRB within the LIGO volume when it is coincident with a GW signal.

\section{Conclusions}

We have shown that the short GRB prompt emission from a realistically structured jet can be detected by observers who are significantly misaligned with respect to the jet axis, provided the short GRB is associated with a GW event that occurs within the LIGO detectability volume. This 


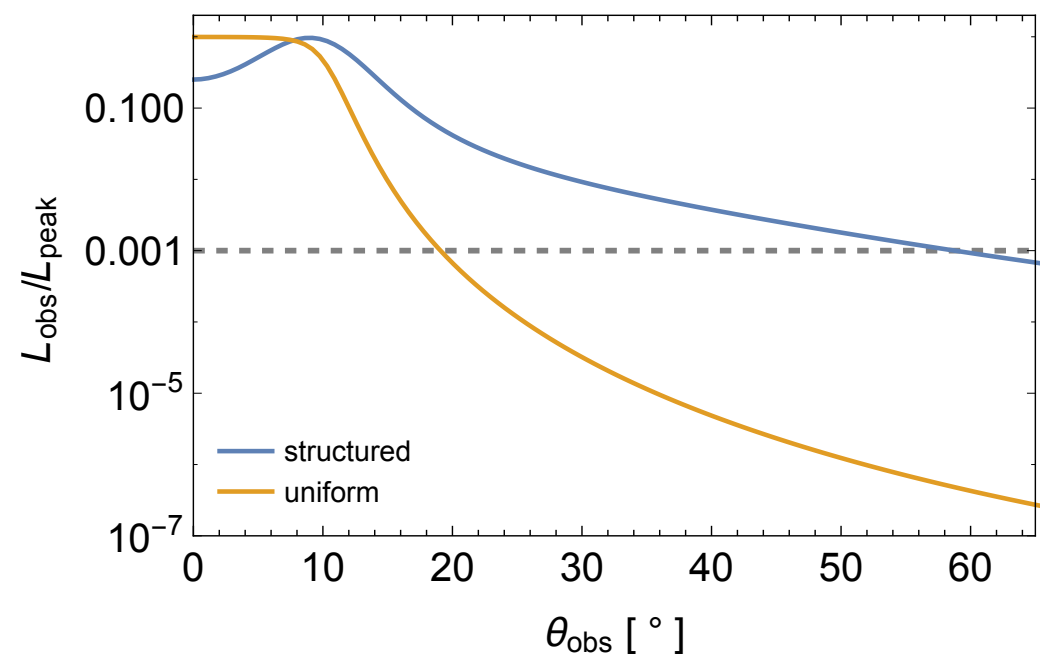

Figure 4: Observed luminosity (normalized to peak) as a function of observer angle, for two different jet models. The blue line shows the emission profile from the structured jet obtained from our simulations output presented in Fig. 3. The orange line shows the emission profile of a uniform jet model with constant $\Gamma=20$ and half opening angle $\theta_{\mathrm{j}}=10^{\circ}$. The dashed gray line indicates the detectability limit of a short GRB within the LIGO volume that is coincident with a GW signal. We find the prompt emission from a structured jet is detectable for a much larger range of observing angles, even though this emission will be faint for off-axis observers, the timing coincidence of the LIGO trigger can help make it a significant detection. In contrast, the emission from the uniform jet is barely detectable beyond the edge of the jet.

can lead to an "off-axis" short GRB as a potential EM counterpart of GW signals. Consequently, we find the rates of detecting such a short GRB can improve substantially when the structure of the jet is taken into account, making the prompt signal a very promising EM counterpart.

\section{References}

[1] W. H. Lee and E. Ramirez-Ruiz. The progenitors of short gamma-ray bursts. New Journal of Physics, 9:17, January 2007. doi: 10.1088/1367-2630/9/1/017.

[2] D. V. Martynov, E. D. Hall, B. P. Abbott, R. Abbott, T. D. Abbott, C. Adams, R. X. Adhikari, R. A. Anderson, S. B. Anderson, K. Arai, and et al. Sensitivity of the Advanced LIGO detectors at the beginning of gravitational wave astronomy. Phys. Rev. D, 93(11):112004, June 2016. doi: 10.1103/PhysRevD.93.112004.

[3] D. Eichler, M. Livio, T. Piran, and D. N. Schramm. Nucleosynthesis, neutrino bursts and gamma-rays from coalescing neutron stars. Nature, 340:126-128, July 1989. doi: 10.1038/340126a0.

[4] E. Nakar. Short-hard gamma-ray bursts. Phys. Rep., 442:166-236, April 2007. doi: 10.1016/j.physrep.2007.02.005.

[5] E. Berger. Short-Duration Gamma-Ray Bursts. ARA\&A, 52:43-105, August 2014. doi: 10.1146/annurev-astro-081913-035926. 
[6] D. Wanderman and T. Piran. The rate, luminosity function and time delay of non-Collapsar short GRBs. MNRAS, 448:3026-3037, April 2015. doi: 10.1093/mnras/stv123.

[7] A. Tchekhovskoy, R. Narayan, and J. C. McKinney. Magnetohydrodynamic simulations of gamma-ray burst jets: Beyond the progenitor star. New Astronomy, 15:749-754, November 2010. doi: 10.1016/j.newast.2010.03.001.

[8] S. S. Komissarov, N. Vlahakis, and A. Königl. Rarefaction acceleration of ultrarelativistic magnetized jets in gamma-ray burst sources. MNRAS, 407:17-28, September 2010. doi: 10.1111/j.1365-2966.2010.16779.x.

[9] K. Sapountzis and N. Vlahakis. Rarefaction wave in relativistic steady magnetohydrodynamic flows. Physics of Plasmas, 21(7):072124, July 2014. doi: 10.1063/1.4891441.

[10] C. F. Gammie, J. C. McKinney, and G. Tóth. HARM: A Numerical Scheme for General Relativistic Magnetohydrodynamics. ApJ, 589:444-457, May 2003. doi: 10.1086/374594.

[11] K. Hotokezaka, K. Kiuchi, K. Kyutoku, H. Okawa, Y.-i. Sekiguchi, M. Shibata, and K. Taniguchi. Mass ejection from the merger of binary neutron stars. Phys. Rev. D, 87 (2):024001, January 2013. doi: 10.1103/PhysRevD.87.024001.

[12] H. Nagakura, K. Hotokezaka, Y. Sekiguchi, M. Shibata, and K. Ioka. Jet Collimation in the Ejecta of Double Neutron Star Mergers: A New Canonical Picture of Short Gamma-Ray Bursts. ApJ, 784:L28, April 2014. doi: 10.1088/2041-8205/784/2/L28.

[13] R. Barniol Duran, A. Tchekhovskoy, and D. Giannios. Simulations of AGN jets: magnetic kink instability versus conical shocks. MNRAS, 469:4957-4978, August 2017. doi: 10.1093/mnras/stx1165.

[14] Adithan Kathirgamaraju, Rodolfo Barniol Duran, and Dimitrios Giannios. Off-axis short GRBs from structured jets as counterparts to GW events. Monthly Notices of the Royal Astronomical Society: Letters, 2017. doi: 10.1093/mnrasl/slx175. URL http://dx.doi.org/10.1093/mnrasl/slx175.

[15] V. Connaughton, E. Burns, A. Goldstein, L. Blackburn, M. S. Briggs, B.-B. Zhang, J. Camp, N. Christensen, C. M. Hui, P. Jenke, T. Littenberg, J. E. McEnery, J. Racusin, P. Shawhan, L. Singer, J. Veitch, C. A. Wilson-Hodge, P. N. Bhat, E. Bissaldi, W. Cleveland, G. Fitzpatrick, M. M. Giles, M. H. Gibby, A. von Kienlin, R. M. Kippen, S. McBreen, B. Mailyan, C. A. Meegan, W. S. Paciesas, R. D. Preece, O. J. Roberts, L. Sparke, M. Stanbro, K. Toelge, and P. Veres. Fermi GBM Observations of LIGO Gravitational-wave Event GW150914. ApJ, 826:L6, July 2016. doi: 10.3847/2041-8205/826/1/L6.

[16] W. Fong, E. Berger, R. Margutti, and B. A. Zauderer. A Decade of Short-duration GammaRay Burst Broadband Afterglows: Energetics, Circumburst Densities, and Jet Opening Angles. ApJ, 815:102, December 2015. doi: 10.1088/0004-637X/815/2/102. 